\title{
Gerodontology - Orodental care for elderly
}

\author{
Vinay Kumar Bhardwaj \\ Department of Public Health Dentistry, H.P. Govt. Dental College and Hospital, Shimla, \\ Himachal Pradesh, India
}

\author{
Address for correspondence: \\ Dr. Vinay Kumar Bhardwaj, \\ Department of Public Health Dentistry, \\ H. P. Govt. Dental College and Hospital, \\ Shimla-171001, Himachal Pradesh, India. \\ E-mail:dr.viney@gmail.com
}

\begin{abstract}
Changing demographics, including an increase in life expectancy and the growing numbers of elderly, has recently focused attention on the need for geriatric dental care. Ageing affects oral tissues in addition to other parts of the human body, and oral health (including oral mucosa, lips, teeth and associated structures, and their functional activity) is an integral component of general health. Oral disease can cause pain, difficulty in speaking, mastication, swallowing, maintaining a balanced diet, not to mention aesthetical considerations and facial alterations leading to anxiety and depression. Certain strategies should be adopted for improving oral health of the elderly, including the management and maintenance of oral conditions, which are necessary for re-establishing effective masticatory function. Oral health is often neglected in the elderly and oral diseases associated with aging are complex, adversely affecting the quality of life. Although majority of oral health problems are not usually associated with mortality, nearly more than half of the deaths due to oral cancer occur at an age of 65 years plus. This review of geriatric dentistry, which is dedicated to geriatric physicians, geriatric dentist and specialists in oral medicine, emphasizes on age-related oral changes in elderly patients and efforts to summarize the effects of aging in hard and soft oral tissues.
\end{abstract}

Key words

Dental care, elderly, gerodontology

\section{INTRODUCTION}

Gerodontology is the delivery of dental care to older adults involving the diagnosis, prevention and treatment of problems associated with normal aging and agerelated diseases as part of an interdisciplinary team with other health care professionals. ${ }^{[1]}$ An overview of the demographics and oral health status of the elderly population of India is presented. India is a vast country with a population of one billion people. Of this, people older than 60 years constitute $7.6 \%$. There are several factors that affect the oral health of elderly. The dentist: population ratio is 1:27,000 in urban areas and $1: 300,000$ in rural areas, whereas $80 \%$ of the elderly population reside in rural India. Forty percent of the elderly live below the poverty line and $73 \%$ are illiterate. Ninety percent of the elderly have no social security and the dependency ratio is 12.26 . Incidence of oral cancer,

\begin{tabular}{|l|l|}
\hline \multicolumn{2}{|c|}{ Access this article online } \\
\hline Quick Response Code: & Website: \\
\cline { 1 - 2 } & www.ejgd.org \\
\cline { 2 - 3 } & \\
\hline
\end{tabular}

which is considered an old-age disease, is highest in India. $13.5 \%$ of all body cancers are oral cancers. Preventive dental care is almost non-existent to the rural masses and is very limited in urban areas. Above all, there is no orientation of dental graduates towards the special needs of the geriatric population. ${ }^{[2]}$

$20^{\text {th }}$ century has witnessed a number of remarkable demographic changes related to health, diseases, longevity and mortality of the population all over the world. By now, one third of the world's elderly population is living in the developing countries and 1 out of 12 persons in the developing countries are over $65 .{ }^{[3]}$ The $20^{\text {th }}$ century witnessed remarkable population ageing with regard to human longevity worldwide and the $21^{\text {st }}$ century is set to carry forward the gains in longevity further, both in the developing and the developed world. This rise in life expectancy is attributed primarily to the substantial reduction in mortality at different stages of life, which has been brought about by improved health care facilities, sanitation, environmental and public health reforms coupled with better hygiene and living conditions. As a result of the increasing life expectancy, the proportion of the elderly in the total population is projected to be around $20 \%$ in India and $32 \%$ in the developed nations by 2050. ${ }^{[4]}$

Keeping this increased life expectancy in mind, the age 
of retirement in many sectors in India is increasing and in some, it has even gone up to 70 years. In some states, the retirement age has not been raised, but this is because of the concerns regarding the resultant job cuts for the younger generation. As per the Government of India's classification, the elderly are those who are 60 years of age and above; these citizens become eligible for varied concessions offered by the government and other agencies. In the developed world, the elderly are those above the age of 65 years. ${ }^{[5]}$

The mouth is referred to as a mirror of overall health, reinforcing that oral health is an integral part of general health. In the elderly population, poor oral health has been considered a risk factor for general health problems. On the other hand, older adults are more susceptible to oral conditions or diseases due to an increase in chronic conditions and physical/mental disabilities. Thus, old people form a distinct group in terms of provision of care. ${ }^{[3]}$ The dental diseases that the elderly are particularly prone to are root caries, attrition, periodontal disease, missing teeth because of earlier neglect, edentulism, poor quality of alveolar ridges, ill-fitting dentures, mucosal lesions, oral ulceration, dry mouth (xerostomia), oral cancers and rampant caries. ${ }^{[2]}$ Many of these are the sequelae of neglect in the early years of life, for example, consumption of a cariogenic diet, lack of awareness regarding preventive aspects, and habits like smoking and/or tobacco, pan, and betel nut chewing. All these problems may increase in magnitude the declining immunity in old age and because of coexisting medical problems. As a result of poor systemic health, the elderly patient often does not pay sufficient attention to oral health. In addition, medications like anti-hypertensives, anti-psychotics, anxiolytics etc. lead to xerostomia, and the absence of the protective influences of saliva in the oral cavity increases the predisposition to oral disease. Financial constraints and lack of family support or of transportation facilities affect access to dental services in later life. ${ }^{[1]}$

However, experiences from developed countries show that the prevalence of chronic disease and high levels of disability in older people can be reduced through health promotion and appropriate prevention strategies designed to improve quality of life. ${ }^{[6]}$ Older people can be a valuable resource; they can contribute to society within their families, communities, and national economies as either a formal or an informal part of the through volunteer work.

\section{WHO AND THE HEALTH OF THE ELDERLY}

In 1995, in response to the global challenges of ageing populations, the World Health Organization (WHO) launched a program on ageing and health. ${ }^{[7]}$ It was designed to advance knowledge about health care in old age through targeted training and research efforts, information dissemination, and policy development. The World Health Report 1998 emphasized the need to strengthen health promotion amongst older people. ${ }^{[8]}$ The health implications of ageing should be better elucidated and understood. Concern for the older members of society is part of the intergenerational relationship that needs to be developed in the $21^{\text {st }}$ century. The young and old must learn to understand each other's differing expectations and requirements. In 2002, WHO issued a document entitled 'Active Ageing - A Policy Framework,' which outlines the essential approaches towards healthy ageing and its framework rests on 3 basic pillars: health, social participation, and security. When risk factors for chronic diseases and functional decline are minimized and protective factors are maximized, people enjoy longer life and higher quality of life. Where labor market, employment, education, health and social policies and programs support full participation of the elderly in socioeconomic and cultural activities, people will continue to make a significant contribution to society as they grow older. When policies and programs address the social, financial, and physical security needs and rights of people as they age, the elderly are ensured protection, dignity and care in the event that they are no longer able to care for themselves. Oral health is an important component of active aging. ${ }^{[7]}$

\section{Oral health problems among elderly}

Globally, poor oral health among older people has particularly been seen in a high level of tooth loss, dental caries experience, high prevalence rates of periodontal disease, xerostomia and oral precancer/cancer. ${ }^{[9]}$ The negative impact of poor oral conditions on daily life is particularly significant among edentulous people. Extensive tooth loss reduces chewing performance and affects food choice; for example, edentulous people tend to avoid dietary fiber and prefer foods rich in saturated fats and cholesterols. ${ }^{[10]}$ Edentulousness is also shown to be an independent risk factor for weight loss ${ }^{[1]}$ and in addition to the problem with chewing, old-age people may have social handicaps related to communication. ${ }^{[12]}$ Moreover, poor oral health and poor general health are interrelated, primarily because of common risk factors; for example, severe periodontal disease is associated with diabetes mellitus, ${ }^{[13]}$ ischemic heart disease, ${ }^{[14]}$ and chronic respiratory disease. ${ }^{[15]}$ Tooth loss has also been linked with increased risk of ischemic stroke ${ }^{[16]}$ and poor mental health. ${ }^{[9]}$

As this concept of Geriatric Dentistry is fairly new to the dental surgeons in India, this initial introduction was necessary. Age does not stop. There are some changes as you grow old. These are called geriatric changes. Geriatric changes take place in the body as well as in the oral cavity also. It is the privilege of dental surgeon to study it well and carry out the necessary treatment of the oral lesions. ${ }^{[17]}$

Oral cavity examination includes following parts of oral 
cavity: mucous membrane, tongue, gingiva, salivary gland, bone, and teeth.

\section{Mucous membrane}

Gums of an adult person are marked shiny and with a stretched appearance. Desired resilience and elasticity are absent. This mucous membrane makes use of dentures very painful. It is important that dental surgeon understands this clinical condition and handles geriatric patient with utmost care. Dental surgeons should be very careful and should avoid any trauma during treatment. ${ }^{[18]}$

Leukoplakia is considered as precancerous condition and tobacco and alcohol could be causative factors. Most of the oral carcinomas are squamous cell carcinomas and common sites are lower lip, tongue, gingivae and floor of the mouth. Oral sub mucous fibrosis (OSMF) is a chronic, insidious, disabling disease involving oral mucosa, the oropharynx and rarely the larynx. It is exclusively reported in elderly Indian population. The disease is characterized by blanching and stiffness of the oral mucosa, trismus, burning sensation in the mouth, hypo mobility of the soft palate and tongue, loss of gustatory sensation and occasionally, mild hearing loss due to blockage of eustachian tube. A variety of etiological factors include capsaicin, betel nut alkaloids, hypersensitivity, autoimmunity and genetic predisposition. ${ }^{[19,20]}$

\section{Tongue}

Dorsum of the tongue shows reddening, atrophy of the papillae at the tip. Tongue may be completely smooth lobulated. These changes bring about an altered taste and decreased appetite. Increased varicosity at the ventral surface is common with elders. It does not call for any treatment unless symptoms appear. Glossodynia or burning tongue, is seen in many adults, sometimes with no apparent clinical picture. It may be vitamin deficiency. Macroglossia, increase in the size of the tongue, in elders is mainly because of loss of tone of muscles of the cheek or expansions or oral cavity as result of loss of teeth. Elderly people are less enthusiastic about proper oral hygiene. They consider other health disorders first on priority bases. They must be explained the consequences, they must be motivated by calling them periodically for checkups.

\section{Salivary glands}

In geriatric patients, salivary flow is decreased causing a condition known as xerostomia. It was presumed that it is an aging process. Now, it is clear that salivary dysfunction is because of certain medicines and systemic diseases. Etiologies that lead to xerostomia are medicines (anti-histaminics, anti-hypertensives, diuretics, antidepressants) and radiation therapy. Unfortunately, these drugs are prescribed to adults very often and hence xerostomia is considered a disease of geriatric patients.

\section{Treatment of xerostomia}

Methods to reduce mouth dryness include avoid dry and bulky foods, spicy or acidic foods and alcoholic beverages. Sucking on sugar-free lozenges or chewing gum to stimulate saliva production, keeping hydrated by sipping water frequently, using a humidifier while sleeping, avoid caffeinated drinks avoid chewing tobacco mouth washes are useful to alleviate oral discomfort. In xerostomia caused by radiation treatment, pilocarpin is the drug preferred and can be consumed for a long period.

\section{Tooth loss}

Edentulism is prevalent among older people all over the world $^{[21]}$ and is highly associated with socio-economic status. Epidemiological studies show that persons of low socio-economic status and individuals with little or no education are more likely to be edentulous than persons of high social class and of high levels of income and education. ${ }^{[9,22]}$ Functional dentitions, as measured by presence of at least 20 natural teeth, are found to be most frequent among elderly of high socio-economic status in contrast to individuals of low socio-economic status. ${ }^{[22]}$ Severe dental caries and periodontal disease are the major reasons for tooth extraction..$^{[9,23-25]}$ Tobacco use is also a risk factor in tooth loss, ${ }^{[23]}$ particularly among people with a high consumption over many years.

\section{Denture-related conditions}

Denture stomatitis is a common oral mucosal lesion of clinical importance in old-age populations. In many cases of denture stomatitis, colonization of yeast to the fitting surface of the prosthesis is observed. Other factors of stomatitis include allergic reaction to the denture base material or manifestations of systemic disease. ${ }^{[26]}$ The prevalence of denture stomatitis correlates strongly to denture hygiene or to the amount of denture plaque. ${ }^{[27,28]}$ Usage of denture at night, ${ }^{[27]}$ neglect of denture soaking at night ${ }^{[28]}$ and use of defective and unsuitable dentures ${ }^{[29]}$ are also risk factors for denture stomatitis, as is tobacco and alcohol consumption. ${ }^{[30]}$ Other major denture-related lesions include denture hyperplasia and traumatic ulcer; their prevalence rates in old-age denture wearers range from $4 \%$ to $26 \% .^{[27,30,31]}$ Denture hyperplasia is particularly frequent in persons with ill-fitting dentures. ${ }^{[12]}$ Both lesions have been observed more often among complete denture wearers than in persons wearing removable partial dentures. ${ }^{[29-31]}$ Moreover, low education, tobacco and alcohol use and infrequent dental visits are factors associated with increased prevalence rate of denture-related lesions. ${ }^{[30]}$

\section{Coronal dental caries and root surface caries}

High prevalence rates of coronal dental caries and root surface caries are found among old-age populations in several countries worldwide. In developing countries, data on dental caries among older people are scarce. A recent survey of 65-74-year-old in Madagascar ${ }^{[32]}$ observed that the mean number of DMFT was 20.2; 
untreated dental caries was high (DT $1 / 4$ 5.3) while the number of restored teeth was low (FT $1 / 40.4$ ). In India, the mean number of decayed teeth has been reported 2.5 in a study. ${ }^{[33]}$ The mean number of decayed and filled root surfaces in older people lies between 2.2 and 5.3 in developed countries ${ }^{[34-36]}$ and in meta-analyzes, the root caries increment has been estimated at 0.47 surfaces per year. ${ }^{[37]}$ The Root caries index, i.e. the number of decayed and filled root surfaces with gingival recession over the number of decayed, filled, and sound root surfaces with recession, ${ }^{[38]}$ was 5.4 in a study of older people in China. ${ }^{[39]}$ In contrast, $12 \%$ of elderly subjects in India had exposed root surfaces, but none experienced root surface caries. ${ }^{[33]}$ The available data worldwide show that dental caries is a major public health problem in older people and is closely linked to social and behavioral factors. ${ }^{[9,21]}$ The pattern is mostly that persons of low income, ${ }^{[40]}$ those who do not visit a dentist regularly, ${ }^{[40,41]}$ do not brush their teeth frequently ${ }^{[41]}$ and smoke, ${ }^{[40]}$ tend to suffer more from coronal and root surface caries.

\section{Oral precancer and oral cancer}

Age-specific rates for cancer of the oral cavity increase progressively with age, most cases occurring in the groups above 60 years. Oral cancer is more common in populations of less developed than of developed countries. ${ }^{[39]}$ The prevalence of leukoplakia and lichen planus in older people ranges from $1.0 \%$ to $4.8 \%$ and $1.1 \%$ to $6.6 \%,{ }^{[31,42-44]}$ respectively. Leukoplakia is more frequent among men while lichen planus is associated with the female gender. ${ }^{[31,43]}$ Tobacco use is the most important determinant of oral cancer and premalignant lesions, ${ }^{[45]}$ including leukoplakia, ${ }^{[31,44]}$ but heavy consumption of alcohol is also a significant factor in relation to these conditions. ${ }^{[4]}$ Socio-economic status such as low levels of education $^{[43]}$ and income ${ }^{[46]}$ is a risk factor for leukoplakia. In contrast, high fruit and vegetable intake are protective factors because of the high content of carotenoids and vitamin C. ${ }^{[47]}$

\section{Oral health care}

Needs and demands for oral health care among the elderly vary across age groups, i.e. the old versus the very old. Oral health systems should effectively address factors that prevent or hinder the older population's access to and use of appropriate services. Some people, for example, experience financial hardship following retirement and the cost or perceived cost of dental treatment may deter them from visiting a dentist. Such barriers to oral health care should be reduced. Age-friendly and prevention-oriented thirdparty payment systems may contribute to effective use of oral health services amongst old age people. In developing countries, barriers to oral health care are particularly high as there is shortage of dental manpower and low priority is allocated to oral health by national health authorities. Affordable oral health care should be organized to ensure adequate early detection, prevention and treatment for all seniors as well as for other age groups. It remains a challenge to health authorities in several developing countries to establish prevention-oriented oral health systems based on the primary health care approach. Community models for outreach service and provision of essential oral health care must be implemented urgently, particularly in low-income communities of Asia and Africa. In a number of developing countries community demonstration projects, based on socio-cultural conditions and focusing on the elderly, are supported or carried out jointly with the WHO.

\section{Provision of oral health care for the elderly}

Elderly people should be provided free oro-dental care and they should be provided treatment on priority in all the health centers. Treatment should be free or on concession to this group of people. Government of Himachal Pradesh, under the project "Muskan,"[48] is providing free dentures to the elderly people above 65 years of age. Such type facility should be provided to the senior citizen throughout the country.

\section{RECOMMENDATIONS}

Establishment of Continuing Dental Education programs on geriatric oral care; inclusion of a geriatric component in undergraduate and postgraduate curricula; initiation of a diploma, certificate and degree courses in geriatric dentistry; research on various aspects of ageing and agerelated oral health problems; provision of preventive and curative treatment for various oral diseases to the elderly. ${ }^{[2]}$

\section{CONCLUSION}

The proportion of older people continues to grow worldwide, especially in developing countries. This, along with an increase in the prevalence of oral disease and non-communicable diseases, will significantly challenge health and social policy planners. The WHO Oral Health Program encourages public health care administrators and decision-makers to design effective and affordable strategies and programs for better oral health and quality of life of the elderly, which are integrated into general health programs. Demonstration projects on oral disease control, health promotion and quality of life improvement should be initiated and evaluated systematically.

\section{REFERENCES}

1. Papas A, Joshi A, Giunta J. Prevalence and intraoral distribution of coronal and root caries in middle-aged and older adults. Caries Res 1992;26:459-65.

2. Shah N. Geriatric oral health issues in India. Int Dent $J$ 2001;51:212- 8 .

3. Schou L. Oral health, oral health care and oral health promotion among older adults: Social and behavioral dimensions. In: Cohen LK, Gift HC, editors. Disease Prevention and Oral Health Promotion. Copenhagen: Munksgaard; 1995.

4. World Health Organization the World Health Report 2003. Shaping the Future. Geneva, Switzerland: WHO; 2003. 
5. World Health Organization. Oral Health Country/Area Profile. Available from: http:/ /www.whocollab.od.mah.se/index.html. [Last cited on 2012 February 24].

6. Puska P, Pietenen P, Uusitalo U. Influencing public nutrition for non-communicable disease prevention: from community intervention to national programme - experiences from Finland. Public Health Nutr 2002;5:245-51.

7. United Nations Population Division. World Population Prospects: The 2002 Revision, New York, NY, USA: United Nations; 2003.

8. World Health Organization. The World Health Report 1998. Life in the $21^{\text {st }}$ Century: A Vision for All. Geneva, Switzerland: WHO; 1998.

9. Walls AG, Steele JG, Sheiham A. Oral health and nutrition in older people. J Public Health Dent 2000;60:304-7.

10. United Nations Population Division. World Population Prospects: The 2002 Revision, New York, NY, USA: United Nations; 2003.

11. Ritchie CS, Joshipura K, Silliman RA. Oral health problems and significant weight loss among community-dwelling older adults, J Gerontol A Biol Sci Med Sci 2000;55:366-71.

12. Smith JM, Sheiham A. How dental conditions handicap the elderly. Community Dent Oral Epidemiol 1979;7:305-10.

13. Shlossman M, Knowler WC, Pettitt DJ. Type-2 diabetes and periodontal disease. J Am Dent Assoc 1990;121:532-6.

14. Joshipura KJ, Rimm EB, Douglass CW. Poor oral health and coronary heart disease. J Dent Res 1996;75:1631-6.

15. Scannapieco F. Role of oral bacteria in respiratory infection. J Periodontol 1999;70:793-802.

16. Joshipura KJ, Hung HC, Rimm EB. Periodontal disease, tooth loss, and incidence of ischemic stroke. Stroke 2003;34:47-52.

17. Papas A, Herman J, Palmer C. Oral health status of the elderly, with dietary and nutritional considerations. Gerodontotogy 1984;3:147.

18. Gupta PC, Mehta FS, Daftary DK. Incidence rates of oral cancer and natural history in a 10-year follow up study of Indian villages. Common Dent Oral Epidemio1 1980;8:287-333.

19. Gangadhran P, Paymaster JC. Leukoplakia-An epidemiologic study of 1504 cases observed at the Tata Memorial Hospital, Bombay, India. Brit J Cancer 1971;25:657-68.

20. Pindborg FF, Mehta FS, Gupta PC. Prevalence of oral submucous fibrosis among 50,915 Indian villagers. Brit J Cancer 1968;22:646-54.

21. Petersen PE. The World Oral Health Report 2003: Continuous improvement of oral health in the $21^{\text {st }}$ century - the approach of the WHO Global Oral Health Programme. Community Dent Oral Epidemiol 2003;31:3-24.

22. Chen M, Andersen RM, Barmes DE. Comparing Oral Health Care Systems. A Second International Collaborative Study. Geneva, Switzerland: WHO; 1997.

23. US Department of Health and Human Services. Oral Health in America: A Report of the Surgeon General. Rockville, MD, USA: National Institutes of Health, National Institute of Dental and Craniofacial Research; 2000.

24. Morita M, Kimura T, Kanegae M. Reasons for extraction of permanent teeth in Japan. Community Dent Oral Epidemiol 1994;22:303-6.

25. Shimazaki Y, Soh I, Koga T. Risk factors for tooth loss in the institutionalized elderly, a six-year cohort study. Community Dent Health 2003;20:123-7.

26. Jeganathan S, Lin CC. Denture stomatitis - a review of the aetiology, diagnosis and management. Aust Dent J 1992;37:107-14.
27. Vigild M. Oral mucosal lesions among institutionalized elderly in Denmark. Community Dent Oral Epidemiol 1987;15:309-13.

28. Shou L, Wight C, Cumming C. Oral hygiene habits, denture plaque, presence of yeasts and stomatitis in institutionalized elderly in Lothian, Scotland. Community Dent Oral Epidemiol 1987; 15:85-9.

29. Fleishman R, Peles DB, Pisanti S. Oral mucosal lesions among elderly in Israel. J Dent Res 1985;64:831-6.

30. Hand JS, Whitehill JM. The prevalence of oral mucosal lesions in an elderly population. J Am Dent Assoc 1986;112:73-6.

31. Jainkittivong A, Aneksuk V, Langlais RP. Oral mucosal conditions in elderly dental patients. Oral Dis 2002;8:218-23.

32. Petersen PE, Razanamihaja N, Poulsen VJ. Surveillance of Oral Health Among Children and Adults in Madagascar. Geneva, Switzerland: WHO; 2004.

33. Thomas S, Raja RV, Kutty R. Pattern of caries experience among an elderly population in South India. Int Dent J 1994;44:617-22.

34. Papas A, Joshi A, Giunta J. Prevalence and intraoral distribution of coronal and root caries in middle-aged and older adults. Caries Res 1992;26:459-65.

35. Winn DM, Brunelle JA, Selwitz RH. Coronal and rootcaries in the dentition of adults in the United States. J Dent Res 1996;75:642-51.

36. Slade GD, Spencer AJ. Distribution of coronal and root caries experience among persons aged 60+ in South Australia. Aust Dent $J$ 1997;42:178-84.

37. Griffin SO, Griffen PM, Swann JL. Estimating rates of new root caries in older adults. J Dent Res 2004;83:634-8.

38. Katz RV. Assessing root caries in populations: the evolution of the root caries index. J Public Health Dent 1980;40:7-16.

39. Lin HC, Schwarz E. Oral health and dental care in modern-day China. Community Dent Oral Epidemiol 2001;29:319-28.

40. Beck JD. The epidemiology of root surface caries. J Dent Res 1990;69:1216-21.

41. Vehkalahti MM, Paunio IK. Occurrence of root caries in relation to dental health behavior. J Dent Res 1988;67:911-4.

42. Steward BW, Kleihues P. World Cancer Report. Lyon, France: WHO International Agency for Research on Cancer; 2003.

43. Reichart PA. Oral mucosal lesions in a representative crosssectional study of aging Germans. Community Dent Oral Epidemiol 2000;28:390-8

44. Pola G, Vallejo MJ, Canel AI. Risk factors for oral soft tissue lesions in an adult Spanish population. Community Dent Oral Epidemiol 2002;30:277-85.

45. Thomas G, Hashibe M, Jacob BJ. Risk factor for multiple oral premalignant lesions. Int $\mathrm{J}$ Cancer 2003;107:285-91.

46. Hashibe M, Jacob BJ, Thomas G. Socioeconomic status, lifestyle factors and oral premalignant lesions. Oral Oncol 2003;39:664.

47. Gupta PC, Hebert JR, Bhonsle RB. Influence of dietary factors on oral precancerous lesions in a population-based casecontrol study in Kerala, India. Cancer 1999;85:1885-93.

48. Project Muskan; Directorate of Dental Health Services Himachal Pradesh. No DDHS-Med-III-(P-HS)-2010-Vol-2.

How to cite this article: Bhardwaj VK. Gerodontology - Orodental care for elderly. Eur J Gen Dent 2012;1:15-9.

Source of Support: Nil, Conflict of Interest: None declared. 